\title{
America's Overview of Superconducting Science and Technology of Ingot Niobium
}

\author{
Gianluigi Ciovati, Peter Kneisel and Ganapati R. Myneni \\ Thomas Jefferson National Accelerator Facility, 12000 Jefferson Avenue, Newport News, VA 23606,
} USA

\begin{abstract}
This contribution will present an overview of the results from R\&D programs in the USA over the past four years towards the development of ingot Niobium as a viable alternative material to fabricate SRF cavities for particle accelerators. Activities at several laboratories and universities include fabrication, surface treatment and RF testing of single- and multi-cell cavities and studies of the thermal, mechanical and superconducting properties of samples from ingots of different purity. Possible advantages of ingot niobium over standard fine-grain (ASTM 6) are discussed and a streamlined treatment procedure to fully exploit those advantages is proposed.
\end{abstract}

Keywords: Superconducting cavities, niobium

PACS: 85.25-j, 74-70.-b

\section{INTRODUCTION}

Since the first successful RF tests of large-grain cavities made from $\mathrm{Nb}$ sheets directly sliced from ingots at Jefferson Lab in 2005, several activities have been pursued by laboratories and universities throughout the world to investigate the applicability of this new technology to the production of superconducting RF (SRF) cavities with performance comparable to that of cavities made of standard high-purity, fine-grain (ASTM 6) Nb. A comprehensive review of these activities up to about 2006 can be found in [1]. The requirements on fine-grain $\mathrm{Nb}$ for SRF cavity production can be found in [2]. Although progress in the surface treatments of fine-grain $\mathrm{Nb}$ cavities over the past decade has pushed their performance close to the theoretical limit of the material [3], large-grain/single-crystal Nb directly sliced from an ingot may have the following benefits over fine-grain material:

- removal of elaborate sheet fabrication steps, such as forging, annealing and rolling

- $\quad$ smooth surfaces achieved by buffered chemical polishing (BCP), which is a cheaper chemical treatment than electropolishing (EP)

- higher thermal stability because of the presence of a phonon peak in the thermal conductivity at about $2 \mathrm{~K}$

- lower residual resistance, resulting in reduced cryogenic losses

- cleaner surfaces by high-pressure rinsing (HPR), resulting in higher values of the surface electric field corresponding to the onset of field emission [4]. 
The possible advantages listed above would result in reduced cost of fabrication, treatment and operation of SRF cavities and the R\&D programs pursued over the past four years are trying to fully investigate those possibilities.

This contribution is organized as follows: the next section will review the progress on fabrication, treatment and RF testing of single- and multi-cell cavities. The next section will review material studies on samples while the following section will propose a streamlined cavity process procedure which could result in higher Q-values (reduced RF losses), which is an important aspect for continuous wave (CW) superconducting Linacs. Finally, some conclusions will be drawn in the final section.

\section{SINGLE- AND MULTI-CELL CAVITIES FABRICATION AND RF PERFORMANCE}

\section{Cavity Fabrication}

Because niobium sheets directly sliced from an ingot have many grains of different orientations with typical area of several $\mathrm{cm}^{2}$, the deep drawing process used to stamp the sheet in a half-cell result in some issues related to the non-uniform grain deformation which can be properly addressed, as discussed in $[5,6]$. As more largegrain cavities have been built in the past few years, some issues with electron-beam welding (EBW) of the equator of dumbbells made of large-grain $\mathrm{Nb}$ has emerged. A full penetration electron beam weld at the equator line from the outside cavity surface is done to join dumbbells or half-cells together to form a cavity. Using the same welding parameters used for fine-grain $\mathrm{Nb}$, the experience at Jefferson Lab showed that a hole was blown during EBW of at least one of the equators during the fabrication of multi-cell cavities (typically 5 to 9 cells). These events, which were much rarer when fabricating single-cell cavities, are not correlated with the particular dumbbell welding sequence but the hole location appears to be near grain boundaries.

The holes had to be repaired by machining the hole region to fit a Nb "plug" which is then electron beam welded. KEK also experienced difficulties with the equator EBW of large-grain cavities but the only company who has built large-grain cavities so far (Research Instruments, Germany) successfully produced eight 9-cell $1.3 \mathrm{GHz}$ cavities with no welding issues [7].

The possibility of cutting $\mathrm{Nb}$ from an ingot with almost any shape or thickness prompted a company (Niowave, USA) to use industrial metal forming processes, other then deep drawing, (for example forging, extrusion, spinning and flow-forming) to fabricate cavity parts with reduced number of components and welds [8].

\section{Single-Cell Cavities}

Extensive RF testing at JLab of $1.3 \mathrm{GHz}$ and $1.5 \mathrm{GHz}$ single-cell cavities made from large-grain $\mathrm{Nb}$ from different manufacturers (CBMM, Brazil, Ningxia, China and W.C. Heraeus, Germany) resulted in excellent, reproducible performances: the average quench field at $2.0 \mathrm{~K}$ from $51 \mathrm{RF}$ tests of 18 different cavities occurred at a peak surface magnetic field $\left(\mathrm{B}_{\mathrm{p}}\right)$ of $140 \pm 14 \mathrm{mT}$ [5]. The residual resistivity ratio 
(RRR) of the material used to fabricate these cavities ranged between 280 and 500 and no correlation between RRR and quench field value was found. The cavity treatments before RF testing are based on material removal by BCP, hydrogen degassing at 600 ${ }^{\circ} \mathrm{C}$ or $800{ }^{\circ} \mathrm{C}$, HPR, baking at $120^{\circ} \mathrm{C}$, post-purification at $1250{ }^{\circ} \mathrm{C}$ with Ti. The cavity treatments are described in greater detail in [5].

Three sets of four additional $1.3 \mathrm{GHz}$ single-cell cavities were built with large-grain $\mathrm{Nb}$ from CBMM, Ningxia and W.C. Heraeus in order to further study the reproducibility of the RF performance. The cavities were subjected to the same treatments consisting of $25-50 \mu \mathrm{m}$ material removal by BCP, heat-treatment at 600 ${ }^{\circ} \mathrm{C} / 10 \mathrm{~h}, 50-90 \mu \mathrm{m}$ BCP, HPR and baking at $120^{\circ} \mathrm{C} / 12 \mathrm{~h}$. The average quench field at 2.0 K measured for the W.C. Heraeus and Ningxia cavities is $147 \pm 19 \mathrm{mT}$ and $141 \pm$ $18 \mathrm{mT}$, consistent with the results from the earlier study [1]. In both cases, the $\mathrm{Q}_{0^{-}}$ value at the quench field is about $1 \times 10^{10}$. RF testing of the cavities built from CBMM material is ongoing.

The RF cavity tests also showed that the onset of the so-called high-field Q-drop, which is a common feature of cavities made of bulk $\mathrm{Nb}$, is shifted to $\mathrm{B}_{\mathrm{p}}$-values of about $110-120 \mathrm{mT}$ compared to about $90 \mathrm{mT}$ for fine-grain cavities treated by BCP [9].

In one case, the RF performance of a $1.5 \mathrm{GHz}$ large-grain single-cell cavity made from CBMM ingot $\mathrm{B}$ niobium showed a steep Q-degradation starting already at $\mathrm{B}_{\mathrm{p}} \cong$ $20 \mathrm{mT}$ [10]. Temperature maps showed few localized hot-spots in the equator region. Samples from the hot-spot regions were cut and different measurement techniques were applied to gain further insight on the possible loss mechanism, as will be discussed in greater detail in the next section.

\section{Multi-Cell Cavities}

Several multi-cell cavities in the GHz range have been built at JLab over the past 4 years:

- two 1.3 GHz 9-cell cavities of the TESLA/ILC shape [11] (LG1 and LG2)

- $\quad$ one $1.3 \mathrm{GHz} 7$-cell cavity of the Low Loss (LL) shape [12]

- two 1.497 GHz 7-cell cavities of the High Gradient shape proposed for the CEBAF Upgrade [13] (HG-A and HG-B)

- two 1.497 GHz 7-cell cavities of the Low Loss shape used for the CEBAF Upgrade [13] (J100-1 and J100-2)

- $\quad$ one 1.497 GHz 5-cell cavity of the High Current (HC) shape [14].

Most of these cavities were built using $\mathrm{Nb}$ from different ingots, named $\mathrm{A}, \mathrm{B}, \mathrm{C}$ and $\mathrm{D}$, provided by CBMM. The RRR of the ingots is $\sim 280$ and the main difference is the Ta content: $~ 800$ wppm in ingots $A$ and $B, \sim 1500$ wppm in ingot $C$ and 1300 wppm in ingot D. As mentioned above, all of these cavities had a one hole burnt through at least one of the equatorial EBW. Pictures of some of the large-grain multicell cavities built at JLab are shown in Fig. 1.

The surface preparation, similar to that of the single-cell cavities, consisted of $\sim 50$ $\mu \mathrm{m}$ material removal by BCP, heat treatment at $600{ }^{\circ} \mathrm{C} / 10 \mathrm{~h}, \sim 50 \mu \mathrm{m}$ BCP, HPR. Cavities J100-2 and LG1 were also baked at $120^{\circ} \mathrm{C}$. Some cavities (HC) were also 
post-purified at $1250{ }^{\circ} \mathrm{C} / 3 \mathrm{~h}$ in a Ti box followed by $\sim 50 \mu \mathrm{m}$ BCP. Some cavities (LG1 and HC) were electropolished, removing about $35 \mu \mathrm{m}$. The best RF test results at $2.0 \mathrm{~K}$ of the $1.3 \mathrm{GHz}$ and $1.497 \mathrm{GHz}$ cavities are shown in Figs. 2 and 3, respectively. The average quench field value of all multi-cell cavities is $100 \pm 18 \mathrm{mT}$. This value is $40 \%$ lower than achieved on single-cells and the standard deviation is about twice as large as that obtained from single-cell cavity tests. One reason for this poorer RF performance can certainly be attributed to the repaired holes at the equator (high magnetic field region). Cavities LG1 and LG2 were tested with a 2-cell thermometry system [15] to detect the quench location, which occurred at the place of the weld repair. In addition, features such as etch pits and "grooves" were observed on the inside cavity surfaces and those might have been caused by not sufficient agitation of the acid during BCP process in the production closed-cabinet system at JLab.

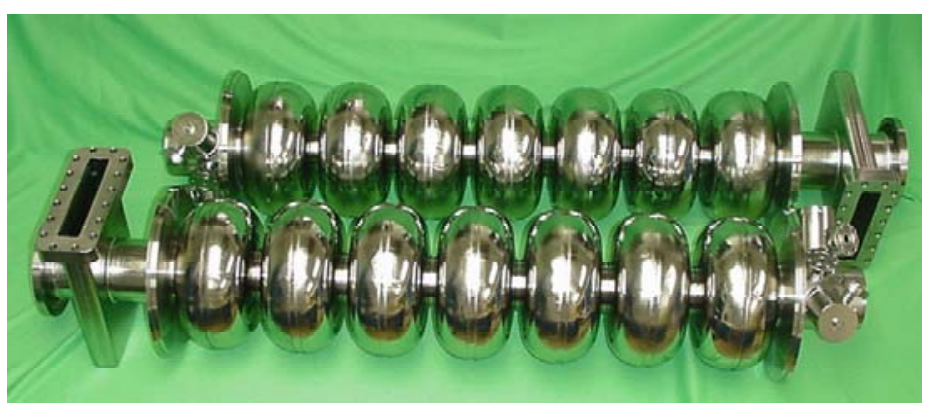

a)

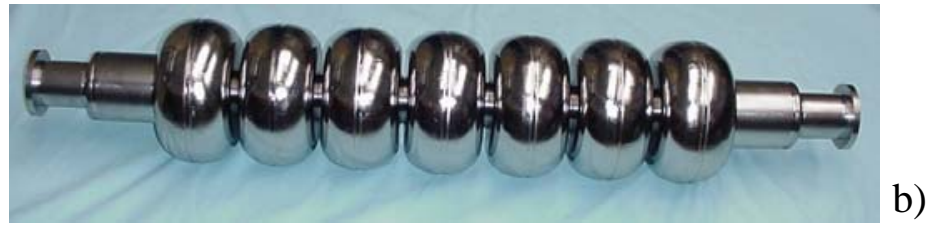

FIGURE 1. Picture of the $1.497 \mathrm{GHz}$, 7-cell cavities J100-1 and J100-2 (a) and of the $1.3 \mathrm{GHz} 7$-cell Low Loss cavity (b) built from large-grain $\mathrm{Nb}$ at Jefferson Lab.

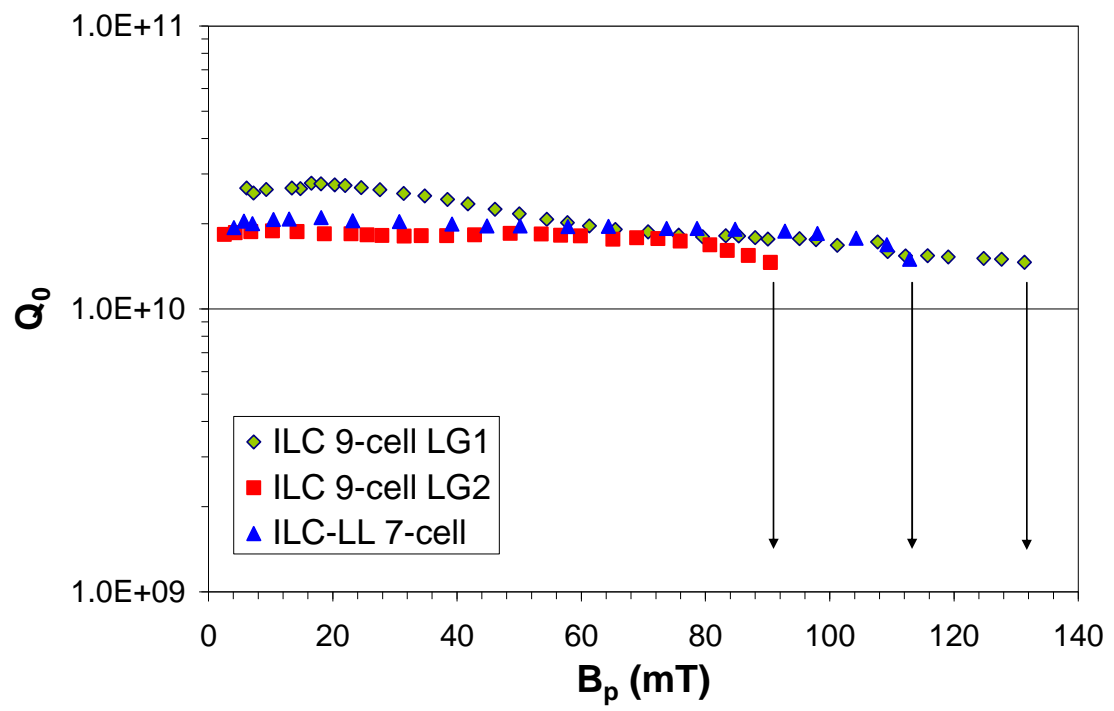

FIGURE 2. Best RF performance measured at $2.0 \mathrm{~K}$ for the three $1.3 \mathrm{GHz}$ multi-cell cavities at JLab. The cavity LG1 was electropolished and baked at $120^{\circ} \mathrm{C} / 48 \mathrm{~h}$. The arrows indicate a quench. 


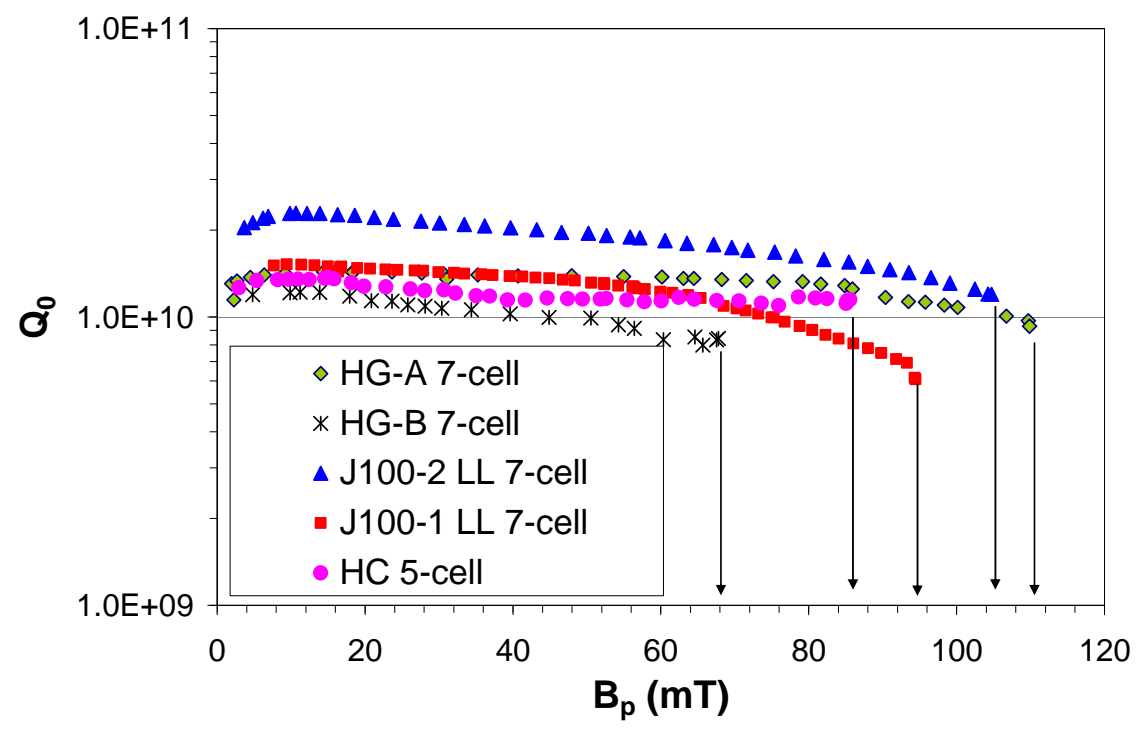

FIGURE 3. Best RF performance measured at $2.0 \mathrm{~K}$ for the five $1.497 \mathrm{GHz}$ multi-cell cavities at JLab. The cavity J100-2 was baked at $120^{\circ} \mathrm{C} / 12 \mathrm{~h}$. The arrows indicate a quench.

Interestingly, the lowest performing multi-cell cavities were built from CBMM ingot $\mathrm{B}$, the same one from which the single-cell with anomalous losses mentioned above was made from. Two other single-cell cavities made from the same material had good performance $\left(\mathrm{B}_{\mathrm{p}, \text { quench }}=112-143 \mathrm{mT}\right)$ [16]. One such multi-cell cavity was the 5-cell, $1.497 \mathrm{MHz}$ HC. The cavity was subjected to several different surface preparation methods which included, besides the ones mentioned in the previous paragraph, barrel polishing (BP) [17, 18]. A summary of the RF test results at $2.0 \mathrm{~K}$ after various preparation procedures in shown in Fig. 4. The quench field did not exceed $95 \mathrm{mT}$. 


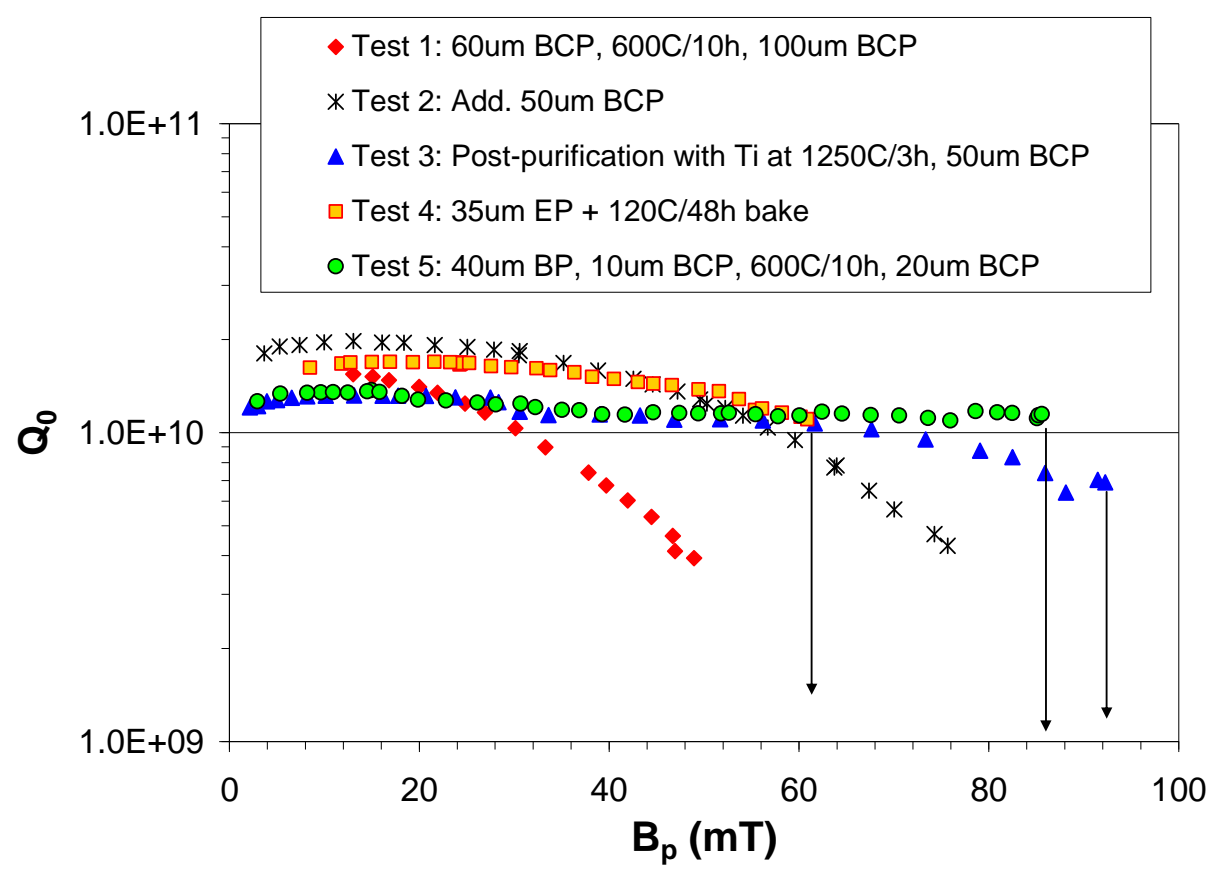

FIGURE 4. RF test results at $2.0 \mathrm{~K}$ for the $1.497 \mathrm{GHz}$, 5-cell HC cavity after different surface preparation processes.

A positive result from the multi-cell cavities was the confirmation of maintaining high $\mathrm{Q}_{0}$-values (greater than $1 \times 10^{10}$ ) up to $\mathrm{B}_{\mathrm{p}}>100 \mathrm{mT}$, as it was measured on singlecell cavities. The values of the residual resistance were deduced from the $\mathrm{Q}_{0}$-values at low field, as described in [19], for the test results shown in Figs. 3 and 4 and are lower than those obtained on fine-grain cavities at the same frequency, as shown in Fig. 5. The values for the fine-grain cavities are the average of several tens of cavities built, processed and tested at JLab: $805 \mathrm{MHz}$ SNS cavities (treated by BCP), $1497 \mathrm{MHz}$ CEBAF refurbishment cavities (treated by BCP) and $1300 \mathrm{MHz}$ ILC cavities (treated by EP) [19].

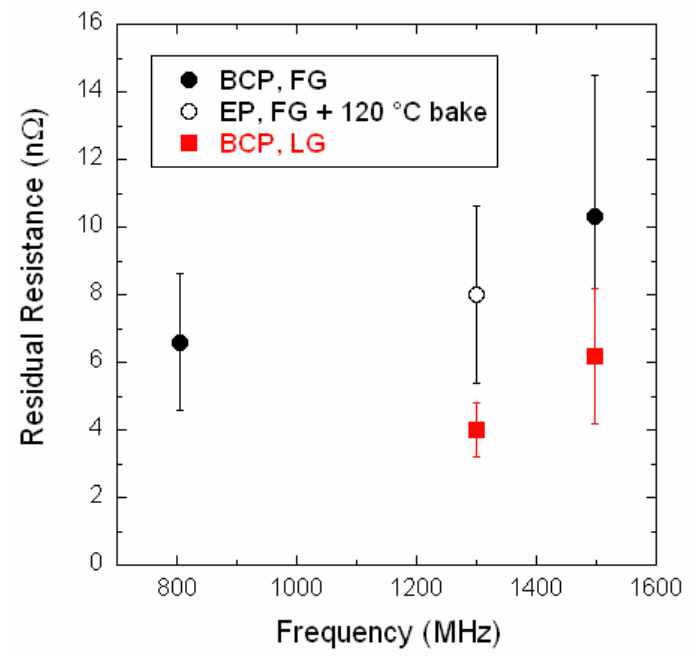

FIGURE 5. Residual resistance values obtained from the low-field $\mathrm{Q}_{0}(2.0 \mathrm{~K})$ for fine-grain (FG) [19] and large-grain (LG) cavities at different frequencies. 


\section{SAMPLE STUDIES}

\section{Samples Cut from Single-Cell Cavity}

Twelve samples were cut by milling from the $1.5 \mathrm{GHz}$ single-cell cavity made from CBMM ingot B niobium which exhibited anomalous RF losses at low fields. Nine samples were from regions of high RF losses ("hot-spots") and three from region of low RF losses (“cold-spot”). Optical microscopy and Secondary Electron Microscope (SEM) images showed many etch pits on the samples' surface, with size 20-80 $\mu \mathrm{m}$ and 2-10 $\mu \mathrm{m}$ in depth as measured by 3D profilometry [10]. Higher density of etch pits correlates with the hot-spot behavior. Nevertheless, the pit distribution is not uniform among the multiple grains but attempts to correlate higher density of pits with a particular grain orientation is not yet conclusive. Magnetic field enhancement at the pits' edges is not sufficient to explain the steep decrease in $Q_{0}$ occurring already at $B_{p}$ $\cong 20 \mathrm{mT}$.

Point Contact Tunneling (PCT) was used at Argonne National Lab to measure the electronic density of states in several regions of the samples at different temperatures and applied DC magnetic field. The measurements showed a large peak in the center of the energy gap in hot-spot rather than cold-spot samples. The temperature and field dependence of the peak could be fitted using the Anderson-Appelbaum theory which is related to the presence of magnetic impurities within the tunnel oxide or at the oxide/metal interface [20].

\section{Measurement of Superconducting Properties}

\section{Jefferson Lab}

Hollow cylindrical samples were machined from a new ingot production series from CBMM. The ingots, which were also labeled A, B, C, and D, have Ta content between $520 \mathrm{wppm}$ and $1320 \mathrm{wppm}$. The samples were mounted in a setup which allows measuring the thermal conductivity as a function of temperature, the magnetization and the penetration depth as a function of temperature and applied external DC magnetic field up to $1 \mathrm{~T}$ [21]. The sample preparation consisted of 180 $\mu \mathrm{m}$ material removal by BCP, $600{ }^{\circ} \mathrm{C} / 10 \mathrm{~h}$ followed by $24 \mu \mathrm{m}$ BCP and baking at different temperatures, between $120^{\circ} \mathrm{C}$ and $160{ }^{\circ} \mathrm{C}$, for $12 \mathrm{~h}$. Although the RRR of the samples from the different ingots ranges between 61 and 146, as obtained from the thermal conductivity value at $4.2 \mathrm{~K}$, measured after $180 \mu \mathrm{m}$ BCP 1:1:1, there was no significant difference in the values of the field of first flux penetration, $\mathrm{H}_{\mathrm{ffp}}$, (the analogous of the lower critical field $\mathrm{H}_{\mathrm{c} 1}$ for a sample with reversible magnetization curve) nor of the upper critical field, $\mathrm{H}_{\mathrm{c} 2}$, measured at $2.0 \mathrm{~K}$. Those values were $\mathrm{H}_{\mathrm{ffp}}=$ $176 \pm 5 \mathrm{mT}$ and $\mathrm{H}_{\mathrm{c} 2}=400 \pm 5 \mathrm{mT}$ [22]. Measurements of the penetration depth at 270 $\mathrm{KHz}$ as a function of the applied DC magnetic field allows measuring $\mathrm{H}_{\mathrm{ffp}}$ and $\mathrm{H}_{\mathrm{c} 2}$ closer to the surface. The data showed:

- $\mathrm{H}_{\mathrm{ffp}}(2.0 \mathrm{~K})$ values of about a $110 \mathrm{mT}$, lower than the bulk value 
- a weak dependence of $\mathrm{H}_{\mathrm{ffp}}$ on RRR

- $\quad$ an increase of $\mathrm{H}_{\mathrm{ffp}}(2.0 \mathrm{~K})$ up to about $130 \mathrm{mT}$, independent of RRR, after 120 ${ }^{\circ} \mathrm{C}$ bake.

These results are summarized in Fig. 6 and discussed in [22] in greater detail. $\mathrm{H}_{\mathrm{ffp}}$ is of particular interest because fluxons entering the Nb surface is a possible cause of the high-field Q-drop observed in SRF cavities [23]. The same samples have been treated by EP, followed by baking at $120^{\circ} \mathrm{C} / 12 \mathrm{~h}$ and the measurement results of the samples' superconducting properties after these treatments are reported in [24].

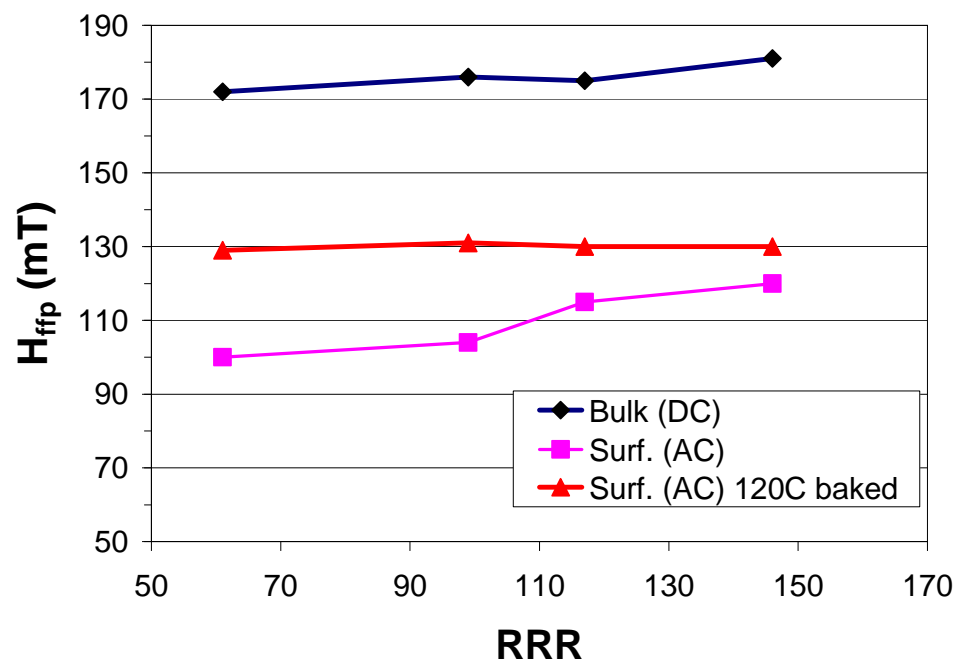

FIGURE 6. Field of first flux penetration obtained from DC magnetization measurements and from AC measurement, before and after baking at $120^{\circ} \mathrm{C} / 12 \mathrm{~h}$, as a function of the RRR of four large-grain samples [20].

It was attempted to measure the quench field in RF of the same cylindrical samples, by inserting them into a pill-box cavity to form a coaxial cavity, excited in the $\mathrm{TE}_{011}$ mode at $3.5 \mathrm{GHz}$. Heating followed by quench was measured at $\mathrm{B}_{\mathrm{p}} \cong 25 \mathrm{mT}$ on the sample and seems to be caused by the small cross section of the samples' channel, so that the RF dissipated power reaches the critical heat flux for He II at rather low RF fields. All these measurements will be repeated on new samples with larger diameter, machined from new ingots from CBMM.

\section{Florida State University/Applied Superconductivity Center}

Bi-crystal samples cut from large-grain $\mathrm{Nb}$ sheets have been studied to understand flux penetration and motion along grain boundaries using different techniques: magneto-optical imaging and DC transport measurements [25, 26]. Both techniques showed that preferential flux penetration at a grain boundary can occur and it is highly sensitive to the orientation between the grain boundary plane and the applied external field. Flux penetration and flux flow along a grain boundary was clearly observed when the applied external field is parallel to the grain boundary plane. 
In addition, a micro Hall probe was used to measure the magnetic field enhancement at a grain boundary step. A field enhancement factor of about 5 was measured at the edge of a grain boundary step with height $\sim 7 \mu \mathrm{m}$ and $10^{\circ}$ slope, relative to the normal direction.

\section{Measurement of Mechanical and Thermal Properties}

Extensive studies of the metallurgical properties of large-grain and single-crystal $\mathrm{Nb}$ samples have been carried out at Michigan State University (MSU) [27]. In particular, stress vs. strain curves were measured on single-crystal samples of different orientation and the results were correlated with the most active slip systems for each particular orientation. In addition, electron backscatter diffraction (EBSD) was used to study the crystallographic properties of single-crystal samples after EBW and showed that welding plastically strained single crystals leads to recrystallization, while it is possible to maintain a single-crystal structure when welding unstrained samples. EBSD studies also showed that Nb sheets sliced from different ingots have different orientation gradient distributions which can impact their formability. Thermal conductivity measurements on large-grain and single-crystal samples were also carried out at MSU and showed the presence of a phonon peak at $2.0 \mathrm{~K}$ [27]. Future work will evaluate the impact of strain and heat treatments on the phonon peak.

Tensile test measurements on single-crystal samples of different orientations were also done at the National Institute of Standards and Technology (NIST) and showed yield stress values between 25 and $40 \mathrm{MPa}$ [28]. Multi-axial straining tests of tricrystal plates were also done and the local strain rate was measured during the test in an attempt to determine the locally active slip systems during the deformation process [29].

Tensile tests at cryogenic temperatures on bi-crystal and single-crystal samples were done at Oak Ridge National Lab. The yield strength increased from about 50-80 $\mathrm{MPa}$ at room temperature to about $560 \mathrm{MPa}$ at $77 \mathrm{~K}$ [30]. Tensile tests of large-grain $\mathrm{Nb}$ samples heat treated at $800{ }^{\circ} \mathrm{C} / 3 \mathrm{~h}$ and $600{ }^{\circ} \mathrm{C} / 10 \mathrm{~h}$ were recently contracted by JLab to St. Louis Test Lab. The yield strength at room temperature was generally greater than $48 \mathrm{MPa}$ and increased up to $512 \mathrm{MPa}$ at $4 \mathrm{~K}$ [31]. At JLab, the evaluation of the cavity design requires the allowable stress to be lower than $2 / 3$ of the measured yield strength under a variety of load conditions [32].

\section{STREAMLINED TREATMENT PROCEDURE FOR LARGE- GRAIN CAVITIES}

Although standard cavity treatment procedures, such as BCP and heat treatment, equally applied to cavities with different grain size (single-crystal, large-grain and fine-grain) did not show significant variations in cavity performance, grain boundaries are still preferred locations for impurity segregation. In particular, oxygen and hydrogen are interstitials which are known to affect significantly the cavity RF performance. In this respect, large-grain niobium may have some advantages over fine-grain because of the reduced grain boundary length. We think that it could be 
possible to develop a streamlined cavity treatment procedure which could lead to $\mathrm{Q}_{0^{-}}$ values at $2.0 \mathrm{~K}$ and $\mathrm{B}_{\mathrm{p}} \cong 90 \mathrm{mT}$ about a factor of 2 greater than typically achieved in fine-grain cavities at the same temperature and RF field, at a reduced cost. The treatment procedure after cavity fabrication is outlined as follows and each step is further discussed in the following paragraph:

- $\quad \sim 80 \mu \mathrm{m}$ material removal by centrifugal barrel polishing (CBP)

- $\quad 20 \mu \mathrm{m}$ material removal by BCP

- heat treatment at $800{ }^{\circ} \mathrm{C} / 3 \mathrm{~h}+120^{\circ} \mathrm{C} / 12 \mathrm{~h}$ in a clean UHV furnace

- $\quad$ surface passivation by depositing a thin nitride layer

- HPR.

CBP is a mechanical material removal method developed and routinely used at KEK [33]. Its main advantage is to remove the damaged layer after forming while obtaining a uniform, smooth finish of the inner cavity surface, particularly in the weld regions. The amount of material removal by CBP and BCP may need to be optimized.

Recent studies of the RF performance of cavities heat-treated at $800{ }^{\circ} \mathrm{C} / 3 \mathrm{~h}+120$ ${ }^{\circ} \mathrm{C} / 12 \mathrm{~h}$, without subsequent chemical etching, showed $25 \%-80 \%$ improvement of the $\mathrm{Q}_{0}(2.0 \mathrm{~K}, 100 \mathrm{mT})$ compared to a baseline test after BCP. The $\mathrm{Q}_{0}$ improvement was due to a reduction, up to $\sim 50 \%$, of the BCS surface resistance combined with low values of residual resistance $(\sim 5 \mathrm{n} \Omega)$ [34]. Samples were heat treated with the cavities and analyzed by Secondary Ion Mass Spectroscopy (SIMS) at North Carolina State University and showed almost two orders of magnitude reduction in hydrogen concentration, compared to the control, BCPed samples [34]. A study in Ref. [35] showed that baking at $140{ }^{\circ} \mathrm{C} / 3 \mathrm{~h}$, besides $120^{\circ} \mathrm{C} / 12 \mathrm{~h}$, can also reduce the high-field Q-drop, while reducing the time the cavity sits in the furnace. The success of this treatment, without following chemical etching, requires a clean, UHV (pressure of $10^{-8}$ mbar at $800{ }^{\circ} \mathrm{C}$ ) furnace. In this respect, JLab is planning on purchasing an induction furnace with separate cavity and furnace vacuum.

In order to avoid the re-absorption of hydrogen and oxygen during the furnace cooldown to room temperature, the inner cavity surface could be passivated with a thin ( 10 nm thick) NbN layer, grown with the cavity inside the furnace at some intermediate temperature $\left(\sim 500{ }^{\circ} \mathrm{C}\right)$. Possible methods to grow the film could be plasma discharge or atomic layer deposition. The natural oxide, $\mathrm{Nb}_{2} \mathrm{O}_{5}$, is also a passivating layer but the growth of a dry oxide in the furnace at high temperature could create a layer contaminated with interstitial oxygen between the oxide and the metal, due to thermal diffusion. Interstitial oxygen is known to suppress superconductivity in $\mathrm{Nb}$ : for example, the critical temperature is reduced by $0.93 \mathrm{~K} / \mathrm{at} . \%$. [36].

The final surface preparation step would be the standard HPR to remove particulates from the inner cavity surface and avoid field emission.

\section{SUMMARY AND OUTLOOK}

Despite its first development in 2006, no large-grain cavity has yet been installed in a cryomodule. This suggests that a significant amount of R\&D work is yet to be done for large-grain ingot $\mathrm{Nb}$ to become an acceptable technology to fabricate SRF cavities 
to be installed in particle accelerators. In the US, cavity fabrication and RF testing in the past 4 years has been limited to JLab. Single-cell cavities consistently achieve $\mathrm{B}_{\mathrm{p}^{-}}$ values of $\sim 140 \mathrm{mT}$, corresponding to accelerating fields of $\sim 32 \mathrm{MV} / \mathrm{m}$ but significantly lower fields were reached in multi-cell cavities, mostly because of problems with EBW. In this respect, the industry has done a better job in fabricating multi-cell cavities without such problems.

A complete set of specifications to order large-grain $\mathrm{Nb}$ from the manufacturers is still missing. Sample studies are helping in reducing the parameter space for grain size, orientation and purity of the material which could lead to reproducible mechanical and superconducting properties.

There are at least two important advantages of large-grain Nb over fine-grain, high RRR, Nb, which at the moment haven't fully realized: lower cost and lower RF losses. In the past few years it became evident that lower cost could be fully realized only if the market for large-grain $\mathrm{Nb}$ will expand significantly. The introduction of possibly cheap slicing techniques [37] pushes towards this direction but the adoption of largegrain $\mathrm{Nb}$ technology for large-scale cavity production projects seems necessary to make this expectation reasonable.

The presence of fewer grain boundaries in large-grain vs. fine-grain $\mathrm{Nb}$ has the potential for lower values of residual resistance, which is supported by some cavity test results. In addition, the presence of a phonon peak in the thermal conductivity at $2.0 \mathrm{~K}$ could improve the thermal stability of the cavity and maintain high $\mathrm{Q}_{0}$-values at high RF fields. The development of a cost-effective treatment procedure, such as the one outlined in this article, which could result in $\mathrm{Q}_{0}$-values significantly higher than what is currently achieved with fine-grain $\mathrm{Nb}$, would fully realize the potential of large-grain cavities.

Clearly, a large number of cavities will have to be built and tested according to specified material properties and treatment procedures to prove their effectiveness. This can only be achieved with strong commitments by many laboratories, not only in the US but throughout the world.

\section{ACKNOWLEDGMENTS}

This manuscript has been authored by Jefferson Science Associates, LLC under U.S. DOE Contract No. DE-AC05-06OR23177. The U.S. Government retains a nonexclusive, paid-up, irrevocable, world-wide license to publish or reproduce this manuscript for U.S. Government purposes.

\section{REFERENCES}

1. P. Kneisel, "Progress on Large Grain and Single Grain Niobium-Ingots and Sheet and Review of Progress on Large-Grain and Single-Grain Niobium Cavities” in Proceedings of the $13^{\text {th }}$ Workshop on RF Superconductivity-2007, edited by J. K. Hao, S. L. Huang and K. Zhao, Peking University, Beijing, China 2009, p. 728-733.

2. W. Singer, A. Brinkmann, D. Proch, and X. Singer, Physica C 386, 379-384 (2003).

3. R. L. Geng, “Overview of High Gradient SRF R\&D for ILC Cavities at Jefferson Lab” in Proceedings of the $14^{\text {th }}$ International Conference on RF Superconductivity-2009, edited by M. AboBakr et al., Helmholtz Zentrum, Berlin, Germany, 2009, pp. 213-217. 
4. A. Dangwal Pandey, G. Müller, D. Reschke, and X. Singer, Phys. Rev. ST Accel. Beams 12, 023501 (2009).

5. P. Kneisel, G. R. Myneni, G. Ciovati, J. Sekutowicz, and T. Carneiro, "Development of Large Grain/Single Crystal Niobium Cavity Technology at Jefferson Lab” in Single Crystal-Large Grain Niobium Technology: Proceedings of the International Niobium Workshop-2006, edited by G. R. Myneni, T. Carneiro and A. Hutton, AIP Conference Proceedings 927, Melville, NY, 2007, pp. 8497.

6. M. Pekeler, J. Schwellenbach, and M. Tradt, "Experiences in Large Grain/Single Crystal Cavity Fabrication"in Single Crystal-Large Grain Niobium Technology: Proceedings of the International Niobium Workshop-2006, edited by G. R. Myneni, T. Carneiro and A. Hutton, AIP Conference Proceedings 927, Melville, NY, 2007, pp. 141-148.

7. W. Singer, "Advances in Large Grain Resonators Activities at DESY, W.C. Heraeus and RI”, these proceedings.

8. T. Grimm, "Plans for Improving Stockpile and Cost of Fine-Grained Niobium and Chipless Forming of Ingot Niobium into Cavity Subassemblies", presentation at the $6^{\text {th }}$ SRF Materials Workshop, National High Magnetic Field Laboratory, Tallahassee, FL, 2010.

9. G. Ciovati, Physica C 441, 44-50 (2006).

10.X. Zhao, G. Ciovati, C. E. Reece, and A. T. Wu, "Surface Topography of Hotspot Regions from a Single Cell SRF Cavity”, in 2009 Particle Accelerator Conference, May 4-8, 2009,Vancouver, Canada, to be published.

11. B. Aune et al., Phys. Rev. ST Accel. Beams 3, 092001 (2000).

12. J. Sekutowicz et al., "Design of a Low Loss SRF Cavity for the ILC" in Proceedings of the 2005 Particle Accelerator Conference, edited by C. Horak, ORNL/SNS, Knoxville, TN, 2005, pp. 3342-3344.

13. J. Sekutowicz et al., "Cavities for JLab's $12 \mathrm{GeV}$ Upgrade” in Proceedings of the 2003 Particle Accelerator Conference, edited by J. Chew, LBNL, Portland, Oregon, 2003, pp. 1395-1397.

14.H. Wang, R. A. Rimmer, and G. Wu, "Elliptical Cavity Shape Optimization for Acceleration and HOM Damping” in Proceedings of the 2005 Particle Accelerator Conference, edited by C. Horak, ORNL/SNS, Knoxville, TN, 2005, pp. 4191-4193.

15. G. Ciovati, R. Flood, P. Kneisel, D. Machie, and M. Morrone, “A 2-Cell Temperature Mapping System for ILC Cavities”, Jefferson Lab Technical Note, TN-08-012, 2008.

16. P. Kneisel, G. R. Myneni, G. Ciovati, J. Sekutowicz, and T. Carneiro, "Performance of Large Grain and Single Crystal Niobium Cavities" in Proceedings of the $12^{\text {th }}$ Workshop on $R F$ Superconductivity, Ithaca, NY, 2005, paper MoP09, available online at: http://www.lns.cornell.edu/public/SRF2005/Proceedings.html

17. P. Kneisel et al., "Preliminary Results from Prototype Niobium Cavities for the JLAB Ampere-Class FEL" in Proceedings of the 2007 Particle Accelerator Conference, edited by I. Andrian et al., Albuquerque, NM, 2007, pp. 2487-2489.

18. R. Rimmer et al., "Recent Progress on High-Current SRF Cavities at JLAB" in Proceedings of the $1^{\text {st }}$ International Particle Accelerator Conference, edited by A. Nodo et al., Kyoto, Japan, 2010, pp. 3052-3054.

19. G. Ciovati, R. Geng, J. Mammosser and J. W. Saunders, "Residual Resistance Data from Cavity Production Projects at Jefferson Lab" in Proceedings of the 2010 Applied Superconductivity Conference, to be published.

20. T. Proslier et al., "Results from Point Contact Tunneling Spectroscopy and Atomic Layer Deposition" in Proceedings of the $14^{\text {th }}$ International Conference on RF Superconductivity-2009, edited by M. AboBakr et al., Helmholtz Zentrum, Berlin, Germany, 2009, pp. 137-143.

21. G. Ciovati et al., "A Coaxial TE011 Cavity and a System to Measure DC and RF Properties of Superconductors" in Proceedings of the $13^{\text {th }}$ Workshop on RF Superconductivity-2007, edited by J. K. Hao, S. L. Huang and K. Zhao, Peking University, Beijing, China 2009, p. 98-102.

22. J. Mondal et al., "Characterization of Ingot Material for SRF Cavity Production" in Proceedings of the $14^{\text {th }}$ International Conference on RF Superconductivity-2009, edited by M. Abo-Bakr et al., Helmholtz Zentrum, Berlin, Germany, 2009, pp. 455-461.

23. A. Gurevich and G. Ciovati, Phys. Rev. B 77, 104501 (2008).

24. A. Dhavale et al., "Effect of Surface Treatment on the Superconducting Properties of Niobium", these proceedings.

25. P. J. Lee et al., "Flux Penetration into Grain Boundaries Large Grain niobium Sheet for SRF Cavities: Angular Sensitivity" in Single Crystal-Large Grain Niobium Technology: Proceedings of the International Niobium Workshop-2006, edited by G. R. Myneni, T. Carneiro and A. Hutton, AIP Conference Proceedings 927, Melville, NY, 2007, pp. 113-120. 
26. Z.-H. Sung et al., "Suppressed Superconductivity on the Surface of SRF Quality Niobium for Particle Accelerating Cavity", these proceedings.

27. T. R. Bieler et al., Phys. Rev. ST Accel. Beams, 13, 031002 (2010).

28. T. Gnäupel-Herold, G. Myneni, and R. Ricker, "Investigations of Residual Stresses and Mechanical Properties of Single Crystal Niobium for SRF Cavities” in Single Crystal-Large Grain Niobium Technology: Proceedings of the International Niobium Workshop-2006, edited by G. R. Myneni, T. Carneiro and A. Hutton, AIP Conference Proceedings 927, Melville, NY, 2007, pp. 48-59.

29. T. Gnäupel-Herold, "Uniaxial and Multiaxial Plastic Deformation of Large Niobium Grains", these proceedings.

30. T. S. Byun, S.-H. Kim, and J. Mammosser, J. Nucl. Mat. 392, 420-426 (2009).

31. G. Cheng (private communication).

32. G. Cheng and M. Wiseman, "C100 Cryomodule Niobium Cavity Structural Analysis”, Jefferson Lab Technical Note, TN-10-001, 2010.

33. T. Higuchi et al., "Centrifugal Barrel Polishing of L-band Niobium Cavities" in Proceedings of the $10^{\text {th }}$ Workshop on RF Superconductivity, edited by S. Noguchi, KEK, Tsukuba, Japan, 2001, pp. 431-432.

34. G. Ciovati et al., Phys. Rev. ST Accel. Beams, 13, 022002 (2010).

35. B. Visentin, J. P. Charrier, Y. Gasser and S. Regnaud, "Fast Argon-Baking Process for Mass Production of Niobium Superconducting RF Cavities" in Proceedings of the 2006 European Particle Accelerator Conference, edited by C. Biscari et al., EPS-AG, Edinburgh, Scotland, 2006, pp. 381-383.

36. W. DeSorbo, Phys. Rev. 132, 107 (1963).

37. K. Saito, F. Furuta, and H. Umezawa, "Multi-wire Slicing of Large Grain Ingot Material”, in Proceedings of the $14^{\text {th }}$ International Conference on RF Superconductivity-2009, edited by M. Abo-Bakr et al., Helmholtz Zentrum, Berlin, Germany, 2009, pp. 467-472. 